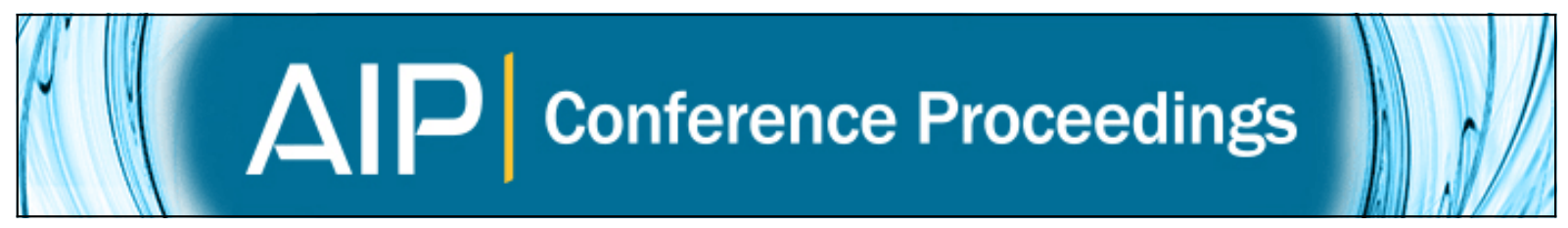

\title{
Flame dynamics in a micro-channeled combustor
}

Taaha Hussain, Christos N. Markides, and Ramanarayanan Balachandran

Citation: AIP Conference Proceedings 1642, 130 (2015); doi: 10.1063/1.4906639

View online: http://dx.doi.org/10.1063/1.4906639

View Table of Contents: http://scitation.aip.org/content/aip/proceeding/aipcp/1642?ver=pdfcov

Published by the AIP Publishing

\section{Articles you may be interested in}

Note: A timing micro-channel plate detector with backside fast preamplifier

Rev. Sci. Instrum. 85, 036103 (2014); 10.1063/1.4867457

Unsteady State Gaseous Flow past a Square Confined in a Micro-channel

AIP Conf. Proc. 1301, 520 (2010); 10.1063/1.3526654

Electroosmotic Dispersion in Micro-Channels and its Implications

AIP Conf. Proc. 1254, 217 (2010); 10.1063/1.3453813

Dissipative Particle Dynamics Simulation of Polymer- and Cell-Wall Depletion in Micro-Channels

AIP Conf. Proc. 1027, 612 (2008); 10.1063/1.2964783

Rarefied Gas Flows in Micro-Channels

AIP Conf. Proc. 663, 800 (2003); 10.1063/1.1581624 


\title{
Flame Dynamics in a Micro-Channeled Combustor
}

\author{
Taaha Hussain*, Christos N. Markides ${ }^{\dagger}$, and Ramanarayanan Balachandran*1 \\ *Department of Mechanical Engineering, University College London, London, UK
${ }^{\dagger}$ Clean Energy Processes Laboratory, Department of Chemical Engineering, Imperial College London, London, UK
}

\begin{abstract}
The increasing use of Micro-Electro-Mechanical Systems (MEMS) has generated a significant interest in combustion-based power generation technologies, as a replacement of traditional electrochemical batteries which are plagued by low energy densities, short operational lives and low power-to-size and power-to-weight ratios. Moreover, the versatility of integrated combustion-based systems provides added scope for combined heat and power generation. This paper describes a study into the dynamics of premixed flames in a micro-channeled combustor. The details of the design and the geometry of the combustor are presented in the work by Kariuki and Balachandran [1]. This work showed that there were different modes of operation (periodic, a-periodic and stable), and that in the periodic mode the flame accelerated towards the injection manifold after entering the channels. The current study investigates these flames further. We will show that the flame enters the channel and propagates towards the injection manifold as a planar flame for a short distance, after which the flame shape and propagation is found to be chaotic in the middle section of the channel. Finally, the flame quenches when it reaches the injector slots. The glow plug position in the exhaust side ignites another flame, and the process repeats. It is found that an increase in air flow rate results in a considerable increase in the length (and associated time) over which the planar flame travels once it has entered a micro-channel, and a significant decrease in the time between its conversion into a chaotic flame and its extinction. It is well known from the literature that inside small channels the flame propagation is strongly influenced by the flow conditions and thermal management. An increase of the combustor block temperature at high flow rates has little effect on the flame lengths and times, whereas at low flow rates the time over which the planar flame front can be observed decreases and the time of existence of the chaotic flame increases. The frequency of re-ignition of successive flames decreases at higher flow rates and increases at higher temperatures. The data and results from this study will not only help the development of new micro-power generation devices, but they will also serve as a validation case for combustion models capable of predicting flame behavior in the presence of strong thermal and flow boundary layers, a situation common to many industrial applications.
\end{abstract}

Keywords: micro-electro-mechanical system, micro-power generation, acetylene-air flames, flame dynamics PACS: 47.61.Fg, 47.70.Pq, 47.80.Jk, 47.70.-n

\section{INTRODUCTION}

The increasing use of Micro-Electro-Mechanical Systems (MEMS) has prompted interest and further research into combustion-based power generation technologies. These systems are of particular interest not only because electrochemical batteries that currently power MEMS devices have short operational lives and are plagued by low power-to-size and power-to-weight ratios, but also because hydrocarbon fuels have a much higher specific energy value compared to some of the top-of-the-range commercial batteries [2]. Though a number of research groups are currently investigating relevant technologies with various computational and experimental approaches, the utilization of such systems in power and heat generation is still in the development stage due to the lack of understanding of the flame dynamics in such devices. Computational models that could potentially simulate the combustion process in micro-channels offer opportunities for the exploration of various design concepts; however these models require high quality experimental data for validation. Unfortunately, due to the difficulties associated with measurements in the harsh combustion environment, and, in small and enclosed spaces, such data is scarce.

Although numerous studies have investigated the flame patterns generated in micro-combustors [3-6], specific knowledge of the dynamics of the flame propagation within the channels is still lacking. This lack of information on the flame dynamics has hindered the successful development of combustion-based MEMS. Flame propagation in a radial micro-channel was studied by Fan et al. [3], where an external heat source was introduced to create a positive temperature gradient in the radial flow direction. This strategy was used to simulate heat recirculation, which in turn

\footnotetext{
${ }^{1}$ Corresponding Author: Dr R Balachandran, r.balachandran@ucl.ac.uk
} 
was successful in stabilizing the flame within the channel over a wide range of operating conditions. Other studies [4,5] either investigated the flame pattern formation, or conducted numerical studies into the dynamics of lean, premixed atmospheric-pressure hydrogen-air flames in planar micro-channels of prescribed wall temperature. These investigations were performed in order to understand the effects of varying reactant inflow velocity and channel height on flame pattern formation. The findings from these investigations were not only specific to the combustor geometry, but were also specific to the fuel-air mixture and flow conditions.

From an examination of the relevant literature, it can be concluded that many micro-combustion related experimental studies involved combustors that relied on strategies involving some form of external heat addition to enable flame stabilization by overcoming the heat losses. Unfortunately, this reliance does not allow a quick cold start. In order to overcome the cold start issue, Kariuki and Balachandran [1] adopted a design that facilitated periodic combustion without flame stabilization. This work also provided quantitative information on the flame dynamics, by reporting on the flame propagation speed for premixed acetylene-air flames. The results indicated that when ignited from a downstream location, a flame propagated inside the channel with a speed that varied with its position. The flame was observed to accelerate upstream, from the exhaust towards the injection manifolds before extinction. The extinction of one flame was followed by the ignition and subsequent propagation of another flame in a repetitive manner.

In this study, the observed flame behavior will be investigated further with the aim of understanding the reported variations in the flame speed. The data presented can be used for the development of a new micro-power generation device, but they also serve as a validation case for combustion models capable of predicting flame dynamics in the presence of strong thermal and flow boundary layers, a situation common to many industrial applications.

\section{METHODS}

\section{Micro-Combustor Design}

Details of the micro-channeled combustor employed in this paper can be obtained from Ref. [1]. Even so, for completeness and clarity, some important information regarding the geometry is provided here. The combustor consists of three parts: (i) a main body that has a cavity for the combustor block, connections for the fuel-air mixture inlet and exhaust gas outlet, and a provision for a glow plug which provides a catalytic surface for re-ignition; (ii) a removable stainless steel combustor block that contains five planar channels of dimension $3 \mathrm{~mm} \times 27 \mathrm{~mm} \times 1.5 \mathrm{~mm}$ (width, length, height), within which the flame propagation occurs; these channels are connected to 'injection slots' $(0.3 \mathrm{~mm} \times 5 \mathrm{~mm} \times 0.3 \mathrm{~mm})$ that allow the flow of reactants into the channels and also act as flame arrestors; and (iii) a UV-grade fused-silica quartz top plate enclosure, which serves as the optical window. Figure 1 shows a schematic of the assembled micro-combustor, including the locations of the inlet, outlet and the catalytic glow plug.

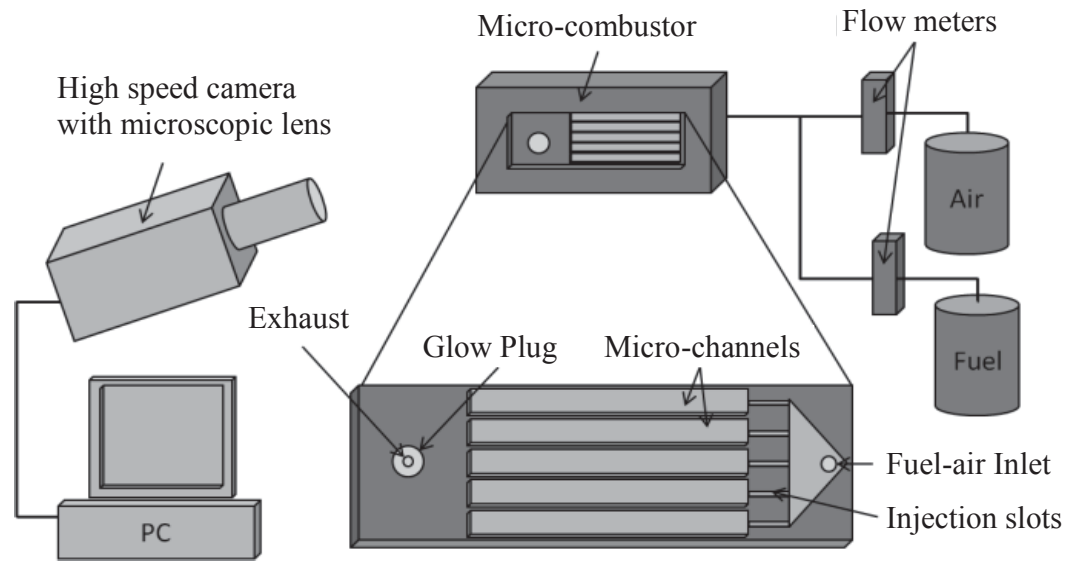

FIGURE 1. Schematic showing high-speed imaging system, flow preparation and micro-combustor arrangement.

The fuel-air mixture was ignited by a catalytic glow plug placed downstream, just before the opening for the exhaust gases. The fuel used was acetylene. The acetylene and air flows were controlled using flow meters, with ranges of $0-0.35$ specific liters per minute $(\mathrm{L} / \mathrm{min})$ for acetylene, and $0-4.0 \mathrm{~L} / \mathrm{min}$ for air, and with an error of 1.25 $\%$ of full scale. In this work, air flow rates between 1.5 and $2.0 \mathrm{~L} / \mathrm{min}$ were used, while the acetylene concentration was varied by using flow rates from 0.13 to $0.22 \mathrm{~L} / \mathrm{min}$. Furthermore, experiments were performed with the combustion block maintained at two different temperatures, $T=40{ }^{\circ} \mathrm{C}$ and $60{ }^{\circ} \mathrm{C}$ in order to investigate the effect of wall temperature (including heat losses) on the dynamics of the flame. 


\section{Flame Propagation}

Once ignited, the flame propagates through the channels and accelerates towards the injection manifold. The flame behavior was recorded with a high-speed camera at a rate of 3,000 frames per second. The recorded images confirmed the overall flame behavior suggested in Ref. [1]. The fuel-air mixture flowing from the injector slots through the micro-channels towards the exhaust manifold is ignited by the glow plug. After ignition, the flame travels inside the channels towards the injection manifold. This travelling flame extinguishes as soon as it reaches the injection ports. After flame extinction, the channels are filled with fresh fuel-air mixture and the process repeats.

It was observed that the flame shape changed during its propagation into the channel. The fast image sequences revealed that the flame enters the channel after ignition and travels as a planar flame for a short distance/time ( $L 1$, t1). Following this stage, the flame passes through the middle region of the channel in chaotic fashion, and finally develops a characteristic "trident" shape (Fig. 2), immediately after which it extinguishes.

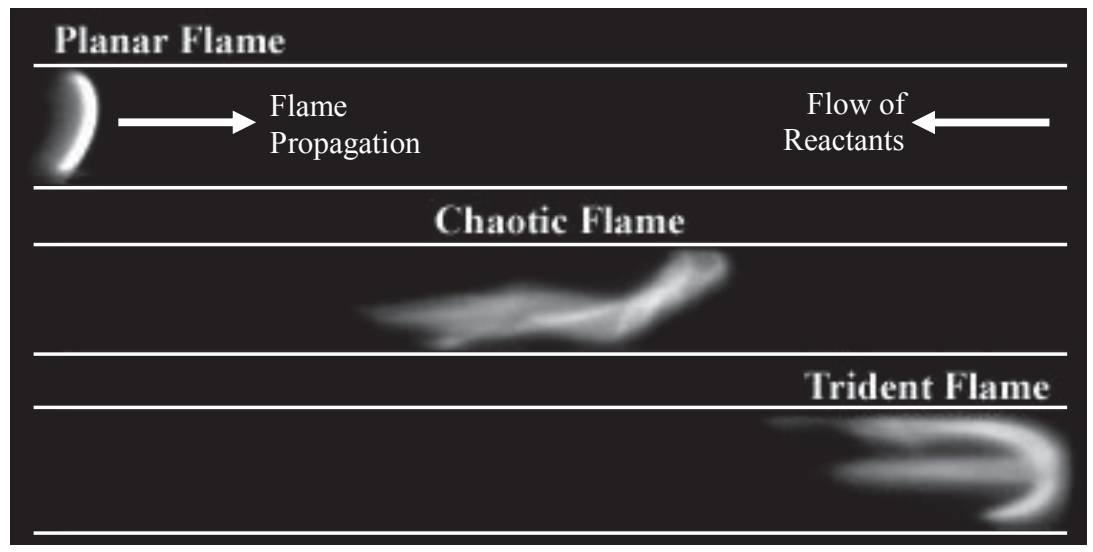

FIGURE 2. Observed flame structure types. Showing flame propagation direction (left-to-right into the channel) and direction of reactant gas flow issued from the injection slots at the far right. The horizontal lines are the channel walls.

From the recorded images and using a MATLAB routine developed in-house, we measured the length from the exhaust side over which the flame remains near-planar, and the corresponding time taken for the flame to travel this length. The time taken for the flame to extinguish after the above transition was also evaluated. The distance travelled by the planar flame will be denoted by $L 1$, and the corresponding time and velocity are $t 1$ and $U 1$, respectively; while the time from this point to extinction and the velocity of the chaotic flame are $t 2$ and $U 2$, respectively. The total time taken from a re-ignition frame to the next consecutive re-ignition sequence will be denoted by $t 3$.

The actual flame propagation speed, $U_{\mathrm{p}}$, is equal to the sum of the absolute velocity of the flame front into the channel, $U_{\mathrm{f}}$, and the absolute velocity of the unburnt reactant gas ahead of the flame, $U_{\mathrm{g}}$, i.e.,

$$
U_{\mathrm{p}}=U_{\mathrm{f}}+U_{\mathrm{g}}
$$

These velocities are illustrated schematically by the diagram in Fig. 3. It can be seen from the expression for $U_{\mathrm{p}}$ in Eq. (1) that when the local flame speed $U_{\mathrm{f}}$ exceeds the local gas velocity $U_{\mathrm{g}}$ the flame propagates upstream through the micro-channel until the flame quenches when it approaches the sudden expansion plane and the high velocity jet.

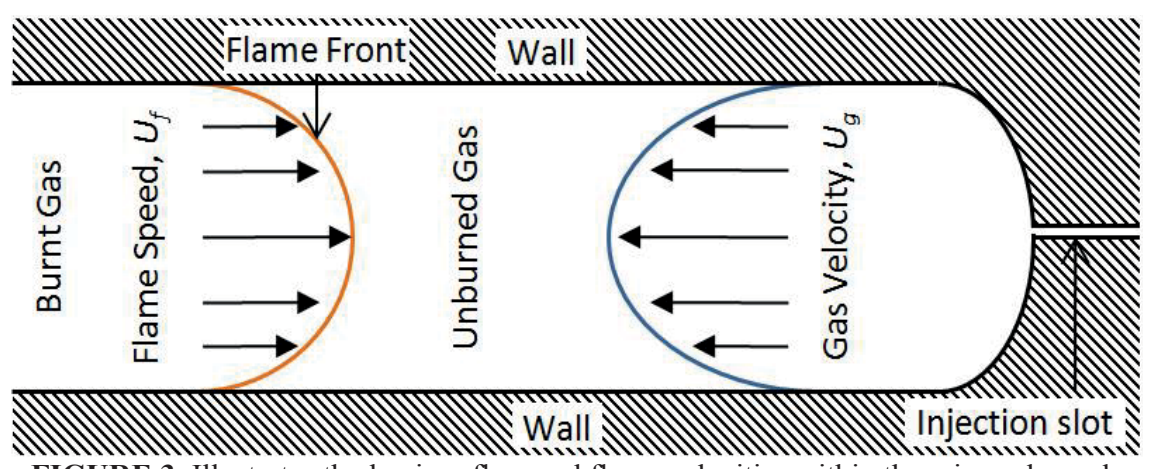

FIGURE 3. Illustrates the laminar flow and flame velocities within the micro-channel. 
The shape of the planar flame as it travels within the channel is expected to be a geometrical reflection of, or at least to be strongly influenced by, the internal gas velocity profile [9-11]. Furthermore, although the local gas velocity at the wall is zero, the resulting shape of the planar flame, especially in close proximity to the wall, will be influenced by the significant temperature difference between the center of the flame and the wall. The wall having a much lower temperature than the center of the flame creates a thermal gradient causing heat and reactive species to diffuse towards the wall, which in turn causes the local laminar flame speed at the wall to be lower than that at the center of the passage [12]. The behavior and stability of the flame is controlled by the heat loss to the walls and the heat release rate of the flame, hence the fuel mixture is varied to achieve a favorable condition for stable operation $[7,13]$.

\section{Computational Flow Field Evaluation}

The micro-channel employed here is a well-researched sudden expansion (backward facing step) type flow passage [1]. In order to visualize qualitatively the flow, we simulated a single micro-channel using a commercial code (ANSYS). The result is shown in Fig. 4. The high velocity jet from the injection slot expands in the microchannel, and a complex recirculation bubble is formed. The flow profile becomes uniform beyond the reattachment points (as shown in the figure).
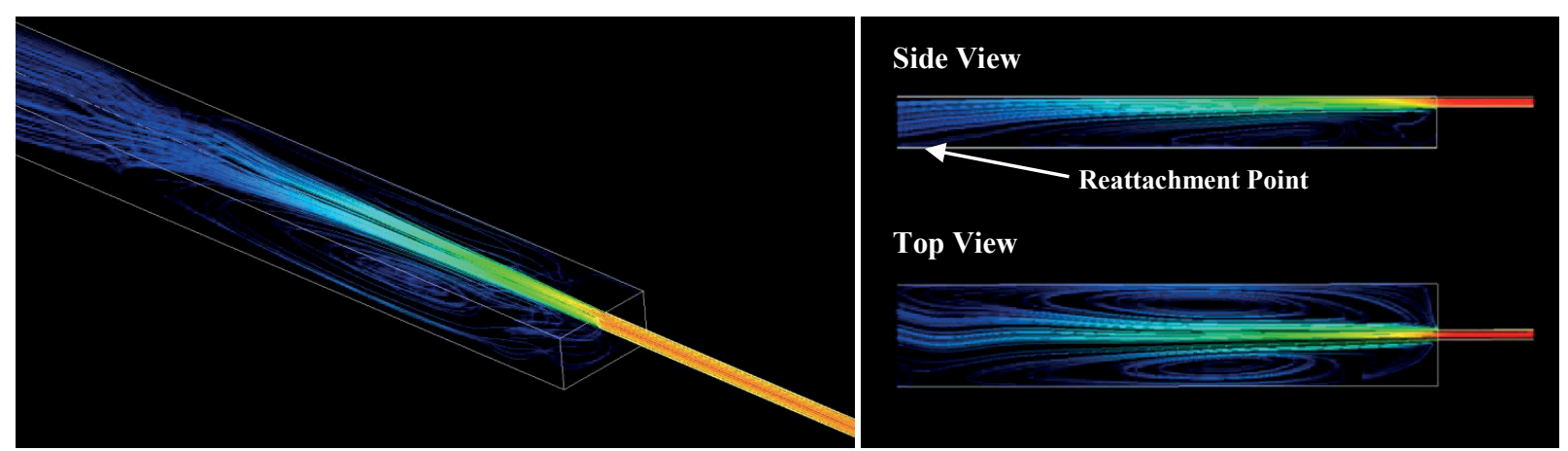

FIGURE 4. Illustration of flow inside the micro-channel using results from ANSYS simulation. Showing the re-attachment point in the side-view insert (see arrow).

This flow behavior correlates with the observed flame shapes. When the flame propagates into the region where the flow is uniform (at the end of the channel), a planar flame shape is observed. In the region of recirculation, the flame is observed to propagate in a chaotic manner. When the flame reaches the high velocity jet and the sudden expansion region, it takes the trident shape (when viewed from above).

\section{RESULTS AND DISCUSSION}

In this section we present the main results from this study, which are presented in Fig. 5 for the planar flame region $(L 1, t 1$ and $U 1)$, Fig. 6 for the events between the emergence of the chaotic flame and extinction ( $t 2$ and $U 2)$, and Fig. 7 for the total time taken from the flame ignition to the ignition of the next one $(t 3)$.

\section{Planar Flame Region}

In Figs. 5(a) and 5(b) we present results for the distance travelled by the planar flame $L 1$ before it develops a chaotic character. From Fig. 5(a) we observe that at low $T=40^{\circ} \mathrm{C}$ there is very little to no change in $L 1$ (within experimental error) as the fuel flow rate, and hence the air-fuel equivalence ratio $\lambda=1 / \varphi$ (here, $\varphi$ is the fuel-air equivalence ratio), is varied. This conclusion holds at both investigated air flow rates $Q_{\text {air }}$ of 1.5 and $2.0 \mathrm{~L} / \mathrm{min}$. By comparing Figs. 5(a) and 5(b) we

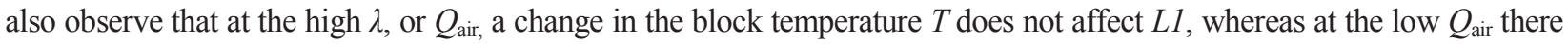
is a clear effect of $T$ on $L 1$. Specifically, at $T=60{ }^{\circ} \mathrm{C}, L 1$ is shorter than its corresponding value at $40{ }^{\circ} \mathrm{C}$, especially at leaner mixtures. In fact, at the lower $Q_{\text {air }}$ and higher $T, L 1$ shows a dependence on $\lambda$, increasing to richer mixtures. The effect of increasing $Q_{\text {air }}$ from 1.5 to $2.0 \mathrm{~L} / \mathrm{min}$ is to almost double $L 1$, at both investigated block temperatures $T$.

Trends for $t 1$ can be seen in Figs. 5(c) and 5(d), while those for $U 1$ can be seen in Figs. 5(e) and 5(f). As with L1, at $Q_{\text {air }}=2.0 \mathrm{~L} / \mathrm{min}, t 1$ and $U 1$ are not strongly affected by $T$. However, when $Q_{\text {air }}$ is reduced to $1.5 \mathrm{~L} / \mathrm{min}$, an increase in $T$ from $40{ }^{\circ} \mathrm{C}$ to $60{ }^{\circ} \mathrm{C}$ leads to a reduction in $t 1$ (of approximately $40-50 \%$ ) and an increase in $U 1$. Furthermore, with $Q_{\text {air }}=1.5 \mathrm{~L} / \mathrm{min}, t 1$ does not change appreciably over the range of investigated $\lambda$, whereas some influence of $\lambda$ can be observed when $Q_{\text {air }}$ is set to $2.0 \mathrm{~L} / \mathrm{min}$, in particular for $t 1$ and $U 1$ at high $T=60^{\circ} \mathrm{C}$. 

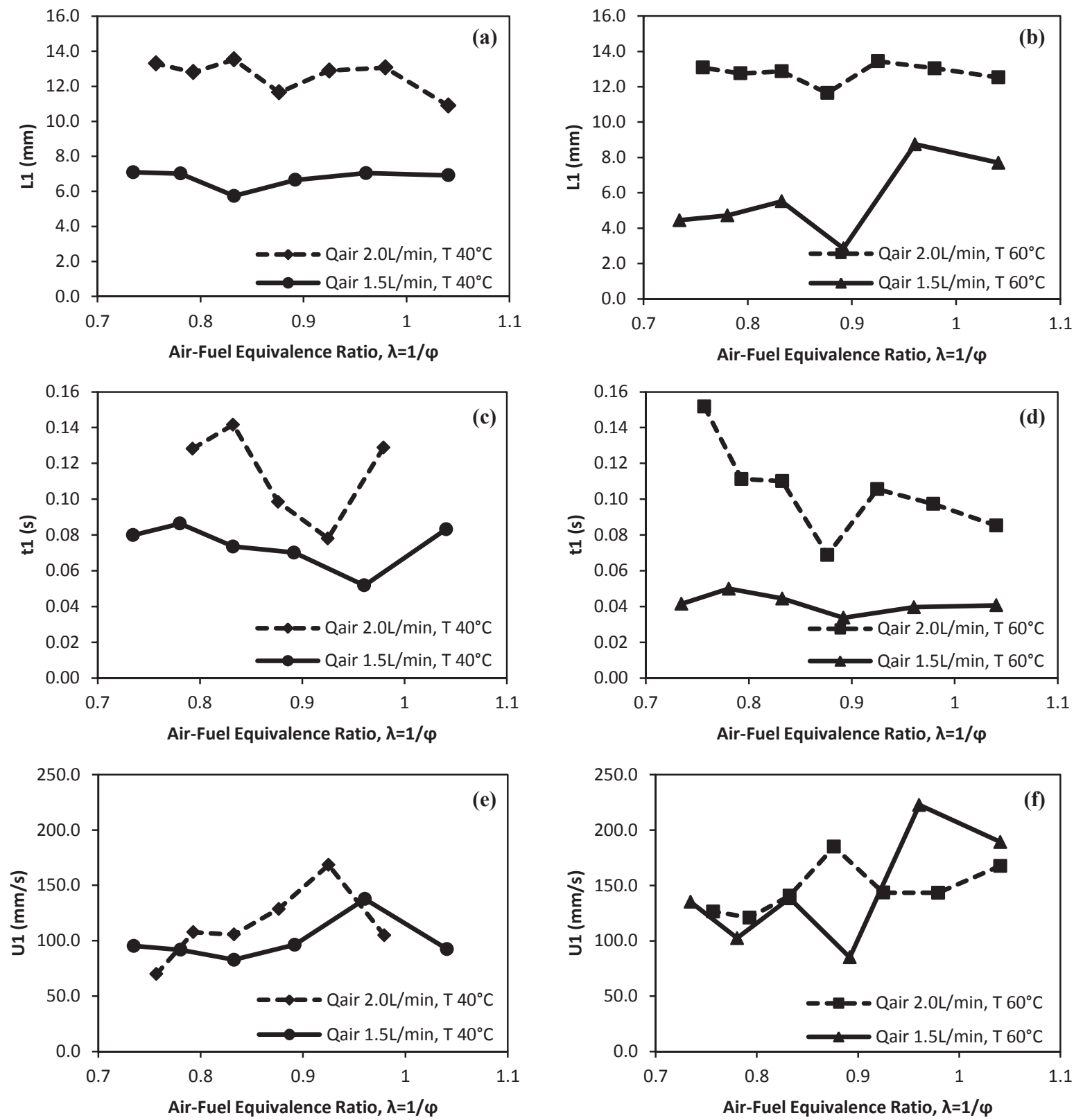

FIGURE 5. Distance $L 1$ at $T=40{ }^{\circ} \mathrm{C}$ (a) and $60{ }^{\circ} \mathrm{C}$ (b) with varying $\lambda$. Time $t 1$ at $T=40{ }^{\circ} \mathrm{C}$ (c) and $60{ }^{\circ} \mathrm{C}$ (d) with varying $\lambda$. Velocity of planar flame $U 1$ at $T=40^{\circ} \mathrm{C}$ (e) and $60{ }^{\circ} \mathrm{C}$ (f) with varying $\lambda$.

Generally, $L 1$ and $t 1$ are greater at the higher air-fuel ratios $\lambda$, or air flow rates $Q_{\text {air }}$ In work conducted by Roy et al. [8], the recirculation bubble generated due to the sudden expansion of the passage was observed to reduce in size and strength as the Reynolds number was increased. Similar results are expected in our current study due to a similar axisymmetric combustor geometry and observed flow geometry within the channel. This reduction in size of the recirculation bubble was explained by fact that the increase in inlet velocity can cause a decrease in the adverse pressure gradient that is responsible for the generation of the bubble. Hence, the increase in $L 1$ at the higher $\lambda$, as seen in Fig 5(a), can be linked to the size of the recirculation bubble, which reduces at the higher inlet velocities.

Referring back to Eq. 1, an increased inlet gas velocity $U_{g}$ should reduce the measured propagation velocity $U_{\mathrm{f}}$, if the actual flame speed $U_{\mathrm{p}}$ remains constant. Looking at Figs. 5(c) and 5(d), the increase in $t 1$ with a change in $Q_{\text {air }}$ from 1.5 to $2.0 \mathrm{~L} / \mathrm{min}$ can be explained by the increase in inlet velocity $U_{\mathrm{g}}$, as the higher gas velocity $U_{\mathrm{g}}$ would have the effect of reducing the measured propagation speed $U_{\mathrm{p}}$ in the stationary experimental frame of reference. This would account for the observed increase in the time taken for the planar flame to propagate $t 1$. Note that also, as discussed earlier, that this increase in $Q_{\text {air }}$ reduces the recirculation bubble size, thus increasing $L 1$, and that the overall propagation velocity of the planar flame $U 1$ increases slightly with increasing $\lambda$ as shown in Fig. 5(e) and 5(f). 


\section{Chaotic and Trident Flame Regions}

Here, we examine the region that begins with the appearance of the chaotic flame, contains the emergence of the trident flame and ends with extinction at the far end of the micro-channel where the injection slots are located. The chaotic flame behavior lasts for much longer than the trident flame, such that this region is dominated and defined mostly by the former.

The formation of the chaotic flame structure observed in the micro-channel is due to the complex recirculation zone created by the sudden expansion of the air-fuel high velocity jet entering the channel from the injection slot. The characteristics and structure of the chaotic flame are quite difficult to predict as they are highly dependent on the size and strength of the recirculation bubble which is quite complex and unsteady in nature. This complexity and unsteadiness increases with an increase in $Q_{\text {air }}$.
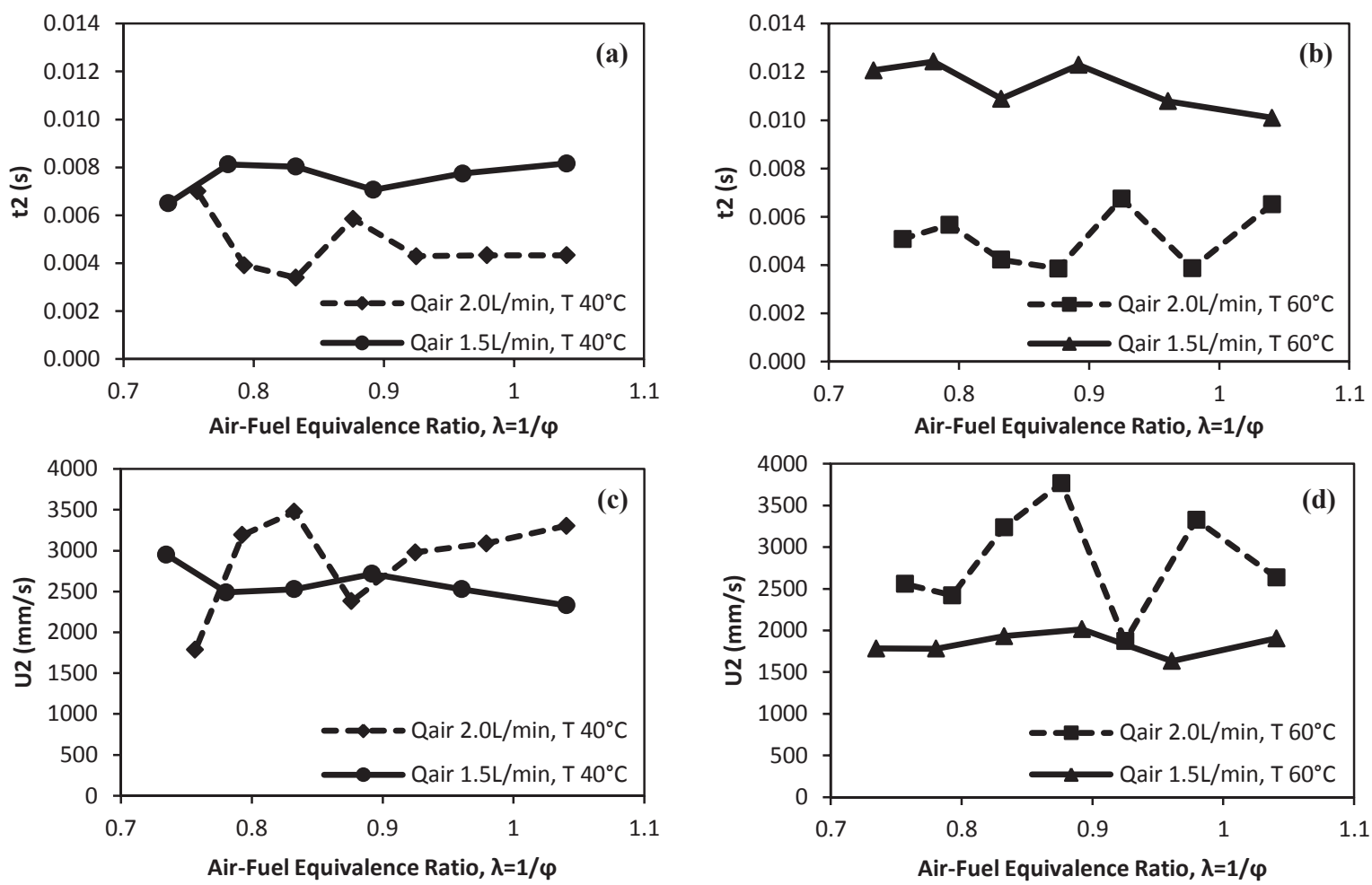

FIGURE 6. Time $t 2$ at $T=40^{\circ} \mathrm{C}$ (a) and $60{ }^{\circ} \mathrm{C}$ (b) with varying $\lambda$. Velocity of chaotic flame $U 2$ at $T=40{ }^{\circ} \mathrm{C}(\mathrm{c})$ and $60{ }^{\circ} \mathrm{C}$ (d) with varying $\lambda$.

Fig. 6 shows experimental data obtained for the chaotic flames observed during operation. In general, the equivalence ratio has very little to no effect on $t 2$. With an increase in $Q_{\text {air, }}$ or $\lambda$, at low temperatures $T=40^{\circ} \mathrm{C}$ there is a reduction in $t 2$, of up to $50 \%$. Similarly, an increase in $\lambda$ at higher $T=60{ }^{\circ} \mathrm{C}$ also leads to shorter $t 2$, though this effect is now even more pronounced (i.e. up to 60\%), compared at the lower $T$. The shorter $t 2$ seen at higher $\lambda$ is in line with our earlier observations concerning the increase in $L 1$ due to the reduction in size and strength of the recirculation bubble with an increase in $\lambda$, and hence the associated reduction in the distance travelled by the chaotic flame. Furthermore, there is no significant effect of the block temperature $T$ on $t 2$ at the high $\lambda$. However, $t 2$ increases considerably (by about 50\%) with an increase in $T$ at the low $\lambda$.

In addition, there is little change in the velocity $U 2$ at low temperatures with varying $Q_{\text {air }}$, or $\lambda$, and finally, at $T=$ $60{ }^{\circ} \mathrm{C}$ there is a reduction in $U 2$ at the lower flow rate. The effect of inreasing $T$ at low $\lambda$ is to reduce $U 2$ by about $20 \%$, while at the higher $\lambda$ is is difficult to conclude that there is a clear effect of $T$.

\section{Re-ignition Frequency}

Finally, Figs. 7(a) and 7(b) show the average time taken for each successive re-ignition $t 3$, while the corresponsing re-ignition frequencies are shown in Figs. 7(c) and 7(d). From this figure what can be understood is that an increase in $Q_{\text {air }}$, or $\lambda$, increases $t 3$ (and reduces the corresponding re-ignition frequency), which indicates that 
at higher inlet velocities the flow is more stable. This ties in with our earlier discussion concerning the increased $L 1$ at the higher $\lambda$, as it takes longer for the planar flame to propogate. Interestingly, the effect of increasing the combustor block temperature $T$ is generally to decrease $t 3$ and to increase the re-ignition frequency, suggesting a destabilizing effect of reduced heat losses. This is an area that requires more careful examination.
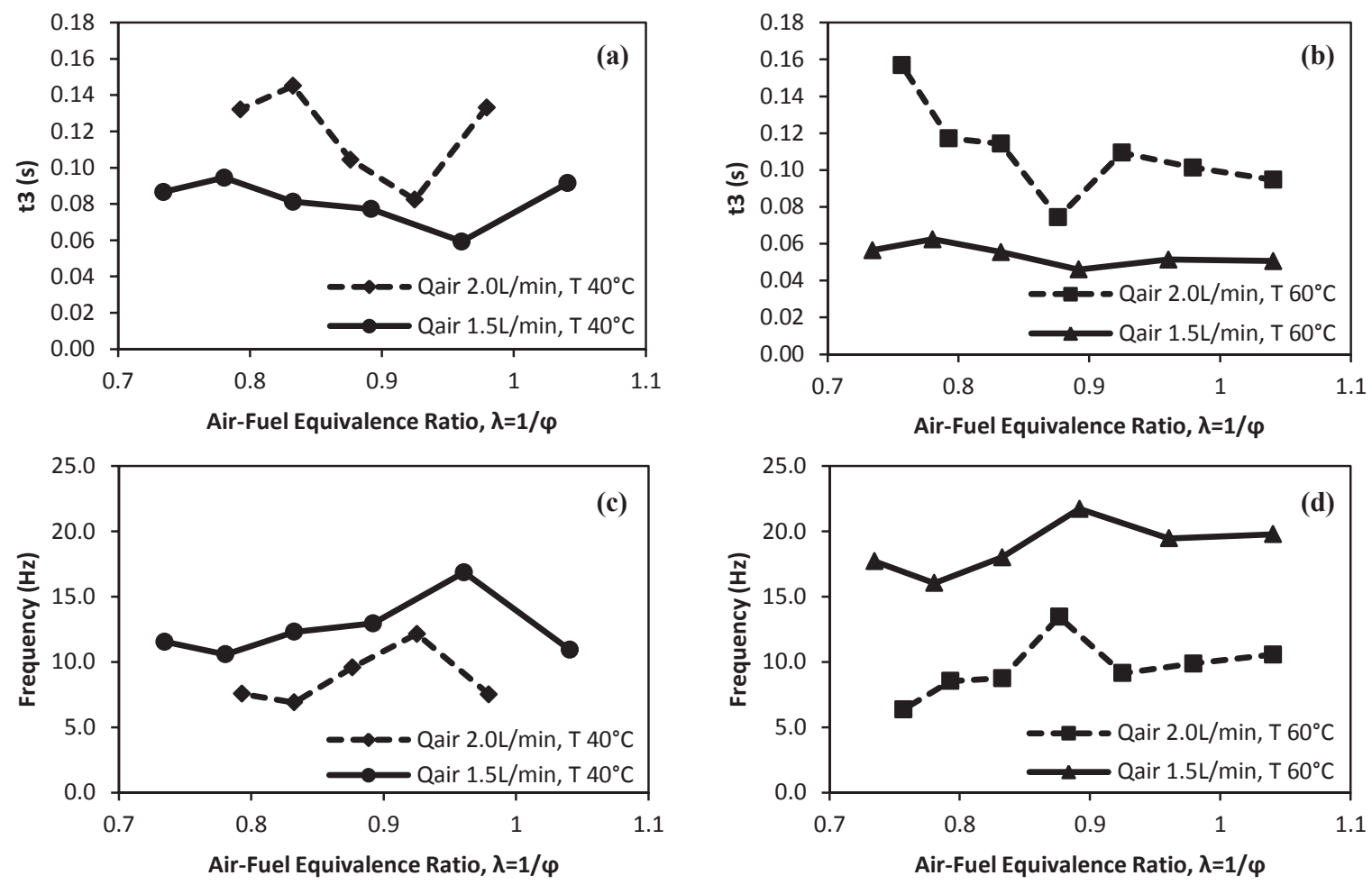

FIGURE 7. Time $t 3$ at $T=40{ }^{\circ} \mathrm{C}$ (a) and $60{ }^{\circ} \mathrm{C}$ (b) with varying $\lambda$. Frequency of sequences at $T=40{ }^{\circ} \mathrm{C}(\mathrm{c})$ and $60{ }^{\circ} \mathrm{C}(\mathrm{d})$ with varying $\lambda$.

\section{CONCLUSIONS}

This paper describes the dynamics of fully premixed acetylene flames in a micro-channeled combustor. Three flame structures were observed. In order of appearance these are: (i) a planar flame at the downstream end of the micro-channel; (ii) an intermediate chaotic flame region; and (iii) an upstream trident flame in the immediate vicinity of the injector slots deep inside the channel, just before the flame extinguishes. The effects of injection velocity, equivalence ratio and temperature of the combustor block on the flame dynamics are investigated. From the results it can be concluded that the inlet velocity plays a major role in the flame propagation within the micro-channel. It was found that at low combustor block temperatures, an increase in air flow rate results in a considerable increase in the length (and associated time) over which the planar flame travels once it has entered a micro-channel, and a significant decrease in the time taken between its conversion into a chaotic flame and its extinction. These findings were also confirmed at higher combustor block temperatures. An increase of the combustor block temperature at high flow rates has little effect on the flame lengths and times, whereas at low flow rates the time over which the planar flame front can be observed decreases and the time of existence of the chaotic flame increases. The frequency of re-ignition of successive flames decreases at higher flow rates and increases at higher temperatures.

The data presented on the flame dynamics within a micro-channeled combustor will not only help in development of a new micro-power generation device, but it will also serve as a validation case for combustion models capable of predicting flame dynamics in the presence of strong thermal and flow boundary layers, a situation common to many industrial applications. Future work will involve understanding the heat transfer characteristic of the microcombustor with periodic heat release as it is complex and is vital for the design of an integrated micro-engine system. Detailed chemical kinetics (wall-chemistry interaction) is required for the accurate prediction of flame behavior in micro-combustors, and so would require detailed chemistry data from advanced diagnostic techniques. 


\section{REFERENCES}

1. J. Kariuki and R. Balachandran, Exp. Therm. Fluid Sci. 34, 330-337 (2010).

2. A. Fernandez-Pello, Proc. Combust. Inst. 29, 883-899 (2002).

3. A. Fan, S. Minaev, S. Kumar, W. Liu and K. Maruta, J. Micromech. Microeng. 17, 2398-2406 (2007).

4. K. Maruta, T. Kataoka, N. Kim, S. Minaev and R. Fursenko, Proc. Combust. Inst. 30, 2429-2436 (2005).

5. G. Pizza, C. Frouzakis, J. Mantzaras, A. Tomboulides and K. Boulouchos, Combust. Flame 152, $433-450$ (2008).

6. C. Miesse, R. Masel, M. Short and M. Shannon, Combust. Theory Model. 9, 77-92 (2005).

7. G. Chen, C. Chen, C. Wu and Y. Chao, Appl. Catal. 332, 89-97 (2007).

8. V. Roy, S. Majumder and D. Sanyal, Int. J. Eng. Sci. Technol. 2, 1569-1574 (2010).

9. H. Najm, P. Paul, C. Mueller and S. Wyckoff, Combust. Flame 113, 312-332 (1998).

10. C. Beyler and F. Gouldin, Proc. Combust. Inst. 18, 1011-1019 (1980).

11. G. Jomaas, X. Zheng, D. Zhu and C. Law, Proc. Combust. Inst. 30, 193-200 (2004).

12. S. R. Turns, "Chapter 8: Laminar Premixed Flames," in An Introduction to Combustion. Concepts and Applications, New York: McGraw-Hill, 2000, pp. 253-304.

13. C. Cadou, T. Leach and G. Jackson, Combust. Theory Model. 10, 85-103 (2006). 